\title{
Specker Sequences Revisited
}

\author{
Jakob Grue Simonsen \\ Department of Computer Science, University of Copenhagen (DIKU) Universitetsparken 1, DK- \\ 2100 Copenhagen $\varnothing$, Denmark \\ simonsen@diku.dk
}

\begin{abstract}
Specker sequences are constructive, increasing, bounded sequences of rationals that do not converge to any constructive real. A sequence is said to be a strong Specker sequence if it is Specker and eventually bounded away from every constructive real. Within Bishop's constructive mathematics we investigate non-decreasing, bounded sequences of rationals that eventually avoid sets that are unions of (countable) sequences of intervals with rational endpoints. This yields surprisingly straightforward proofs of certain basic results from constructive mathematics. Within Russian constructivism, we show how to use this general method to generate Specker sequences. Furthermore, we show that any nonvoid subset of the constructive reals that has no isolated points contains a strictly increasing sequence that is eventually bounded away from every constructive real. If every neighborhood of every point in the subset contains a rational number different from that point, the subset contains a strong Specker sequence.
\end{abstract}

\section{Introduction}

In 1949, E. Specker exhibited a strictly increasing, bounded sequence of rationals that was constructive, but did not converge to any constructive real number [Spe49], thus showing that the classical BolzanoWeierstrass Theorem is provably false in Russian constructive mathematics (RUSS), and unprovable in Bishop's constructive mathematics (BISH). A folklore result was that not only did these "Specker sequences" $\left(r_{n}\right)_{n \in \mathbb{N}}$ exist, but there are sequences that are "strongly" Specker: An algorithm can be defined in Russian mathematics that yields, for each constructive real $x$, a pair $(\epsilon, k) \in \mathbb{Q} \times \mathbb{N}$ such that $\left|x-r_{n}\right| \geq \epsilon$ for $n \geq k$, i.e. it is possible to give a constructive witness (in the form of a lower bound) to the fact that $\left(r_{k}\right)_{k \in \mathbb{N}}$ is bounded away from each constructive real. O. Aberth [Abe80] and D. Bridges and F. Richman [BR87] provided proofs of this, the Richman/Bridges proof using Cantor's middle-third set. A method, apparently first employed by K.-I Ko [Ko91] in recursive mathematics, can be used to construct such sequences from a sequence of open intervals covering the constructive reals.

This paper makes the following contributions:

1. We show that Ko's observations can be used to examine both the existence of strong Specker sequences and other results by considering sequences of rational numbers strongly avoiding socalled countably open sets, i.e. countable unions of intervals with rational endpoints. In particular, we may derive well-known results within Bishop's brand of constructivism and the Russian ditto.

2. We show, within RUSS (specifically BISH $+\mathrm{CPF}$ - the axiom that the set of partial functions from the naturals to the naturals is countable), that every nonvoid subset of the constructive reals without isolated points contains a strictly increasing sequence that strongly avoids the constructive reals. If every neighborhood of every point in the subset contains a rational number distinct from that point, the subset contains a strong Specker sequence.

Computer scientists should note that RUSS is essentially recursive mathematics (or "computable analysis") with some minor differences that do not surface in this paper. We refer the reader to [BR87] for a treatment of the various schools of constructivism. 


\section{Preliminaries}

We expect the reader to have a working knowledge of BISH, cf. [BB85, BR87], and of either Russian constructive mathematics or computable analysis [Abe80, Ko91, Wei98]; the well-known Blum-ShubSmale framework for computable analysis [BCSS97] is quite different from the aforementioned notions and will not be treated here. Terminology will be that of constructive mathematics; the reader with background in computer science will thus be well-advised to interpret every statement ("there is X") in this paper as "there is a program computing X" and "countable" as "recursively enumerable". Definitions of standard concepts from classical mathematics, e.g. convergence of sequences and (sequential) continuity carry over to the constructive setting mutatis mutandis, unless otherwise noted.

We begin with some notation:

Definition 2.1 (BISH) The least element of $\mathbb{N}$ is 1 . We set $\mathbb{N}_{0} \triangleq\{0\} \cup \mathbb{N}$. The set $\mathbb{Q}$ denotes $\{p / q: p \in \mathbb{Z}, q \in \mathbb{N}\}$. Observe that comparisons and the standard arithmetical operations on this set are finite procedures. The set of nonvoid open intervals on the constructive real line with endpoints in $\mathbb{Q}$ is denoted by $\mathbb{I}^{\mathbb{Q}}$. If $I, J_{1}, \ldots, J_{n}$ are intervals with endpoints in $\mathbb{Q}$, then $I \backslash\left(J_{1} \cup \cdots \cup J_{n}\right)$ is either empty, or is a finite union of disjoint elements of $\mathbb{I}^{\mathbb{Q}}$; we define $\mu\left(I \backslash\left(J_{1} \cup \cdots \cup J_{n}\right)\right)$ to be 0 in the first case, and the total length of the elements of the disjoint, finite union otherwise. The symbol $\mathbb{R}$ denotes the set of (constructive) reals, i.e. the set of sequences $\left(a_{n}\right)_{n \in \mathbb{N}}$ of elements of $\mathbb{Q}$ such that $\left|x_{n}-x_{m}\right|<1 / m+1 / n$ for all $m, n \in \mathbb{N}$.

We now introduce countably open sets and covers:

Definition 2.2 (BISH) A countably open set is a set $U=\bigcup_{j \in \mathbb{N}} I_{j}$ such that $\left(I_{j}\right)_{j \in \mathbb{N}}$ is a sequence of (not necessarily disjoint) elements of $\mathbb{I}^{\mathbb{Q}}$, called a generator of the set. Let $\epsilon \in \mathbb{R}_{+}$. A generator $\left(I_{j}\right)_{j \in \mathbb{N}}$ is said to be $\epsilon$-enclosed if, for all $n \in \mathbb{N}$ we have $\sum_{j=1}^{n}\left|I_{j}\right|<\epsilon$. It is said to be $\epsilon$-sharp (or just sharp, if $\epsilon$ is unimportant) if $\sum_{j \in \mathbb{N}}\left|I_{j}\right|$ is convergent with limit $\epsilon$. A set $A \subseteq \mathbb{R}$ is said to have an $\epsilon$-cover if there is a countably open set $U=\bigcup_{j \in \mathbb{N}} I_{j}$ with $\sum_{j \in \mathbb{N}}\left|I_{j}\right|<\epsilon$ and $A \subseteq U$.

Observe that a single countably open set may have enclosed, sharp, non-sharp, and non-enclosed generators.

Example 2.3 Simple examples of covers and covered sets are:

1. (BISH) The Cantor Set $C \triangleq\left\{\sum_{n=1}^{\infty} c_{n} 3^{-n}: c_{n} \in\{0,2\}\right.$ for each $\left.n\right\}$ has an $\epsilon$-sharp cover for all $\epsilon \in \mathbb{R}_{+}$.

2. (RUSS) A well-known construction [KL57, TZ62, BR87] yields, for each $\epsilon \in \mathbb{R}_{+}$, a (non-sharp) $\epsilon$-cover of $\mathbb{R}$ (often called a singular cover in the literature). Thus, $\mathbb{R}$ will have (outer) measure 0 in any naïve constructive measure theory.

Definition 2.4 (BISH) Let $U$ be a countably open set. A sequence $\left(r_{k}\right)_{k \in \mathbb{N}}$ of reals is said to be $U$-avoiding if it does not converge to any element of $U$. It is said to be strongly $U$-avoiding if, for any $u \in U$, there are $\delta \in \mathbb{Q}_{+}$and $m \in \mathbb{N}$ such that $\left|r_{j}-u\right| \geq \delta$ for all $j>m$. A (strongly) $\mathbb{R}$-avoiding sequence is called (strongly) divergent. A strictly increasing, bounded sequence of elements of $\mathbb{Q}$ that is (strongly) $\mathbb{R}$-avoiding is called (strongly) Specker.

E. Bishop (and subsequently proponents of K. Weihrauch's Type II computable analysis) used the notion of an operation $M \rightrightarrows N$ to denote a "finitely specified procedure" that takes an element $x \in M$ to an element of $N$ with the proviso that different specifications of $x$ could lead to different elements of $N$ (in Type II computable analysis such "operations" are called "multi-valued functions"). Thus, we could have rephrased the notion of strongly $U$-avoiding sequence to mean existence of an operation $F: U \rightrightarrows \mathbb{Q}_{+} \times \mathbb{N}$ such that $F(u)=(\delta, m)$ implies $\left|r_{j}-u\right| \geq \delta$ for all $j>m$.

\section{The $M^{<}$and $M \leq$ Constructions}

We no present two simple means of constructing monotone sequences of rationals that avoid countably open sets. 
Definition 3.1 (BISH) Let (]$p_{j} ; q_{j}[)_{j \in \mathbb{N}}$ be a generator, and let $p \in \mathbb{Q}$ and $d \in \mathbb{Q}_{+}$. Then $M^{<}\left(p,(] p_{k} ; q_{k}[)_{k \in \mathbb{N}}, d\right)$ is the strictly increasing sequence $\left(r_{n}\right)_{n \in \mathbb{N}}$ of elements of $\mathbb{Q}$ defined by setting $r_{0} \triangleq p$, and defining $r_{n}$ for $n>0$ as follows: let $s \triangleq r_{n-1}+d \cdot 2^{-n}$; if $\left.s \notin \bigcup_{j=1}^{n}\right] p_{1} ; q_{1}\left[\right.$, then set $r_{n} \triangleq s$. Otherwise, let $r_{n}$ be the least $q_{i} \in\left\{q_{1}, \ldots, q_{n}\right\}$ such that $s<q_{i}$ and $\left.q_{i} \notin \bigcup_{j=1}^{n}\right] p_{1} ; q_{1}[$.

It is immediate that the sequence thus constructed is bounded below by $p$. If there is a $q \in \mathbb{Q}$ such that $\sum_{j=1}^{n}\left|I_{j}\right|<q$ for all $n \in \mathbb{N}$, then the sequence is clearly bounded above by $p+q+d$. Further, it is clear that a variant, $M \leq$, of $M^{<}$that only produces a non-decreasing sequence of rationals can be constructed by letting $d=0$. Ko [Ko91], and Hertling and Weihrauch [HW98] observe that one may also construct a non-decreasing sequence by considering some element $r \in] p_{1} ; q_{1}[\cap \mathbb{Q}$ and letting, for each $n \in \mathbb{N}, r_{n}$ be the the right endpoint of the maximal interval subset of $\bigcup_{j=1}^{n} I_{j}$ that contains $r$.

Proposition 3.2 (BISH) Let $A=\left\{x_{1}, x_{2}, \ldots\right\} \subseteq \mathbb{R}$ be countable, and $\epsilon \in \mathbb{Q}_{+}$. Then $A$ is covered by a countably open set with an $\epsilon$-sharp generator (]$p_{j} ; q_{j}[)_{j \in \mathbb{N}}$, such that, for all $j \in \mathbb{N}$, we have $p_{j}+2^{-(j+2)} \epsilon<x_{j}<q_{j}-2^{-(j+2)} \epsilon$.

Proof. For each $x_{j}$, find $p, q \in \mathbb{Q}$ such that $\left.x_{j} \in\right] p ; q\left[\right.$ and $q-p=2^{-(j+1)} \epsilon$. Set $p_{j} \triangleq p-2^{-(j+2)} \epsilon$ and $q_{j} \triangleq q+2^{-(j+2)} \epsilon$. Clearly, $\sum_{k \in \mathbb{N}}\left(q_{j}-p_{j}\right)=\epsilon$.

The following lemma shows that the $M^{<}$and $M \leq$ constructions can be used to create sequences that strongly avoid countably open sets:

Lemma 3.3 (BISH) Let $U$ be the countably open set generated by (]$p_{k} ; q_{k}[)_{k \in \mathbb{N}}$, let $p \in \mathbb{Q}, d \in \mathbb{Q}_{+}$, and let $\left(r_{m}\right)_{m \in \mathbb{N}}$ be shorthand for the sequence $M^{<}\left(p,(] p_{j} ; q_{j}[)_{j \in \mathbb{N}}, d\right)$. Then, for any $x \in U$, there are $n \in \mathbb{N}$ and $\delta \in \mathbb{Q}_{+}$such that, for all $m>n,\left|r_{m}-x\right| \geq \delta$.

Proof. Since $\left.x \in \bigcup_{k \in \mathbb{N}}\right] p_{j} ; q_{j}[$, there exists an $n \in \mathbb{N}$ such that $x \in] p_{n} ; q_{n}[$, and hence $\delta \in \mathbb{Q}$ such that $p_{k}+\delta<x<q_{j}-\delta$.

We can clearly replace $M^{<}$by $M \leq$ in the lemma.

\subsection{Applications of Enclosed Generators}

As a first application, we derive a few well-known results:

Proposition 3.4 The following hold:

1. (BISH) For any countably open set $U \subset[0 ; 1]$ with an enclosed generator $\left(I_{j}\right)_{j \in \mathbb{N}}$ such that $I_{j} \cap I_{j^{\prime}}=$ $\emptyset$ for $j \neq j^{\prime}$, there exists a (continuous, but not necessarily uniformly so) surjection $f:[0 ; 1] \longrightarrow$ $[0 ; 1[$ such that $f(x)>0$ if $x \in U$ and $f(x)=0$ if $x \in \mathbb{R} \backslash U$.

2. (RUSS) Existence of strong Specker sequences: There exists a strictly increasing sequence $\left(r_{n}\right)_{n \in \mathbb{N}}$ of elements of $\mathbb{Q}$ such that for each $x \in \mathbb{R}$, there is $N \in \mathbb{N}$ and $\delta>0$ satisfying $\left|x-r_{n}\right| \geq \delta$ whenever $n \geq N$.

3. (RUSS/CLASS) For each $\epsilon \in \mathbb{Q}_{+}$, there is a continuous map $f:[0 ; 1] \longrightarrow[0 ; 1[$ such that the classical measure of the set $\{x \in[0 ; 1]: f(x)=0\}$ is at least $1-\epsilon$, but for all $x \in \mathbb{R}, f(x) \neq 0$.

Proof. We proceed as follows:

1. Write $\left.I_{j} \triangleq\right] p_{j} ; q_{j}\left[\right.$, for all $j \in \mathbb{N}$. Let $t_{j}(x)$ be a continuous map from $[0 ; 1]$ to $\mathbb{R}$ that maps $\left[p_{j} ; q_{j}\right]$ onto $\left[0 ; 1-j^{-1}\right]$, vanishes outside $] p_{j} ; q_{j}\left[\right.$. Let $f(x) \triangleq \sum_{j \in \mathbb{N}} t_{j}(x)$. If $x \in U$, there exists an $n \in \mathbb{N}$ such that $x \in I_{n}$. Hence, $f(x)>0$, and by disjointness of the $I_{j}, f(x)<1$. If $x \in \mathbb{R} \backslash U$, we clearly have $f(x)=0$. Continuity follows by disjoint of the $I_{j}$.

2. Use Lemma 3.3 on the countably open set of Example 2.3.2.

3. Use the first part of the proposition and the countably open set of Example 2.3.2. 
A version of Part 1 of the above specialized to the countably open set of Example 2.3.2 with $\epsilon=1 / 4$ was used by Specker to demonstrate [Spe59] the existence of a function on [0;1] that attained its maximum on a set of (classical) measure $>3 / 4$, but did not do so for any element of $\mathbb{R}$.

Since the union of any two countably open sets is countably open, we may combine the countably open set covering $\mathbb{R}$ with any other countably open set, and thus obtain:

Corollary 3.5 (RUSS) For any countably open set, $U$, there is a Specker sequence that is strongly U-avoiding.

\subsection{Applications of Sharp Generators}

Up to this point, we have considered how to construct sequences that avoid countably open sets, and showed within RUSS that the classical limit is not necessarily an element of $\mathbb{R}$. A tantalizing question is in what cases the classical limit of such a sequence $i s$ an element of $\mathbb{R}$. It turns out that sharp generators can yield (constructive) reals specified by increasing sequences; to prove this, we first need the following lemma.

Lemma 3.6 (BISH) Let $J$ be a closed interval with endpoints in $\mathbb{Q}$, and let $\left(I_{j}\right)_{j \in \mathbb{N}}=(] p_{j} ; q_{j}[)_{j \in \mathbb{N}}$ be a sharp sequence such that $\sum_{j \in \mathbb{N}}\left(q_{j}-p_{j}\right)<|J|$. Then there is a sequence of closed intervals $J_{0} \supseteq \cdots \supseteq J_{i} \supseteq \cdots$ with endpoints in $\mathbb{Q}$ such that:

1. $J_{i} \subseteq J$,

2. $\left|J_{i}\right| \geq q / 2^{i}$, and

3. $\mu\left(J_{i} \backslash\left(\bigcup_{j=1}^{n_{i}} I_{j}\right)\right)>q / 4^{i}$ (we make the convention that $\bigcup_{j=1}^{0} I_{j}$ is void).

Proof. The proof is by induction on $i$.

Define $J_{0} \triangleq J$ and $n_{0} \triangleq 0$. Observe that $\mu\left(J_{0} \backslash\left(\bigcup_{j=1}^{n_{i}} I_{j}\right)\right)=\left|J_{0}\right|>q$, thus finishing the base case.

The inductive case proceeds as follows: Assume that $i \geq 1$ and that the claim holds for $i-1$. By the induction hypothesis, $\mu\left(J_{i-1} \backslash\left(\bigcup_{j=1}^{n_{i-1}}\right)\right)>q / 4^{i-1}$. We have:

$$
\begin{aligned}
\mu\left(J_{i-1} \backslash \bigcup_{j=1}^{n_{i}} I_{j}\right) & =\mu\left(\left(J_{i-1} \backslash \bigcup_{j^{\prime}=1}^{n_{i-1}} I_{j^{\prime}}\right) \backslash \bigcup_{j=n_{i-1}+1}^{n_{i}} I_{j}\right) \\
& >q / 4^{i-1}-\sum_{j=n_{i-1}+1}^{n_{i}}\left|I_{j}\right| \\
& \geq q / 4^{i-1}-\sum_{j=n_{i-1}}^{\infty}\left|I_{j}\right| \\
& \geq q / 4^{i-1}-q / 4^{i} \\
& =3 q / 4^{i}
\end{aligned}
$$

Write $\left.J_{i-1}=\right] a_{i-1} ; b_{i-1}\left[, d=\left(a_{i-1} ; b_{i-1}\right)\right.$, let $d \triangleq\left(a_{i-1}+b_{i-1}\right) / 2$, and let $\left.A_{1} \triangleq\right] a_{i-1} ; a_{i-1}+d[\backslash$ $\left(\bigcup_{j=1}^{n_{i}} I_{j}\right)$, respectively $\left.A_{2} \triangleq\right] a_{i-1}+d ; b_{i-1}\left[\backslash\left(\bigcup_{j=1}^{n_{i}} I_{j}\right)\right.$.

By the above computation, one of $\mu\left(A_{1}\right)>3 / 2 \cdot q / 4^{i}$ and $\mu\left(A_{2}\right)>3 / 2 \cdot q / 4^{i}$ must be true - recall that all arithmetic and the comparisons are performed in $\mathbb{Q}$. If $\mu\left(A_{m}\right)>3 / 2 \cdot q / 4^{i}$ (for some $m \in\{1,2\}$ ), we set $J_{i} \triangleq A_{m}$. We clearly have $\left|J_{i}\right|=\left|J_{i-1} / 2\right| \geq q / 2^{i}$ and $\mu\left(J_{i} \backslash \bigcup_{j=1}^{n_{i}} I_{j}\right)>3 / 2 \cdot q / 4^{i}>q / 4^{i}$, concluding the proof.

We now have:

Theorem $3.7(\mathrm{BISH})$ Let $J$ be a closed interval with endpoints in $\mathbb{Q}$, and let $\left(I_{j}\right)_{j \in \mathbb{N}}=(] p_{j} ; q_{j}[)_{j \in \mathbb{N}}$ be a sharp sequence such that $\sum_{j \in \mathbb{N}}\left(q_{j}-p_{j}\right)<|J|$. Then there exists a non-decreasing sequence $\left(r_{n}\right)_{n \in \mathbb{N}}$ of elements of $\mathbb{Q} \cap J$, convergent in $J$, that is strongly $\bigcup_{j \in \mathbb{N}} I_{j}$-avoiding (hence an element of $\cap_{j \in \mathbb{N}}\left(J \backslash I_{j}\right)$ ). 
Proof. Let $q \in \mathbb{Q}_{+}$be such that $|J|>q+\sum_{j \in \mathbb{N}}\left(q_{j}-p_{j}\right)$. For each $i \in \mathbb{N}_{0}$, sharpness enables us to find $n_{i} \in \mathbb{N}$ such that $\sum_{j=n_{i}}^{\infty}\left|I_{j}\right|<q / 4^{i+1}$.

Let $\left(J_{i}\right)_{i \in \mathbb{N}}$ be the sequence of Lemma 3.6. We can find the least element, $r_{i}$, of $\mathbb{Q}$ in $J_{i} \backslash\left(\bigcup_{j=1}^{n_{i}} I_{j}\right)$ in a finite number of computations involving only arithmetic in $\mathbb{Q}$, and the sequence $\left(r_{i}\right)_{i \in \mathbb{N}}$ is clearly non-decreasing. Furthermore, for $m, m^{\prime}>N$, we have $\left|r_{m}-r_{m^{\prime}}\right| \leq\left|J_{N}\right|=2^{-N}$, i.e. $\left(r_{n}\right)_{n \in \mathbb{N}}$ is Cauchy, hence an element, $x$, of $\mathbb{R}$. In addition, for $n>n_{i}$, we have $\left.r_{n} \notin \bigcup_{j=1}^{n_{i}}\right] p_{j} ; q_{j}\left[\right.$. Thus, for $u \in \bigcup_{j \in \mathbb{N}} I_{j}$, we can find $m \in \mathbb{N}$ and $q^{\prime} \in \mathbb{Q}_{+}$such that $p_{m}+q^{\prime}<u<q_{m}-q^{\prime}$. We can find a suitable $n_{k}$ with $n_{k}>m$, in which case $\left|r_{i}-u\right|>q$ for $i>n_{k}$, and we thus have $x \in \cap_{j \in \mathbb{N}}\left(J \backslash I_{j}\right)$, since $x \in\left(J \backslash I_{j}\right)$ for all $j \in \mathbb{N}$.

We have tacitly used the Axiom of Countable choice in the proof. In the theorem, the requirement that $\left(I_{j}\right)_{j \in \mathbb{N}}$ be sharp cannot be omitted due to the cover of $\mathbb{R}$ of Example 2.3.2. Consider, now, the following well-known result by N. Greenleaf:

Theorem 3.8 (BISH) Let $J$ be a bounded, closed interval and $\left(I_{j}\right)_{j \in \mathbb{N}}$ a sequence of bounded, open intervals such that $\sum_{j \in \mathbb{N}}\left|I_{n}\right|$ converges to a sum less than $|J|$. Then $\cap_{j \in \mathbb{N}}\left(J \backslash I_{j}\right)$ is nonvoid.

Greenleaf's Theorem can be inspected in the light of countably open sets: If $J$ is a closed, bounded interval and $\left(I_{j}\right)_{j \in \mathbb{N}}$ a sequence of bounded, open intervals that is $|K|$-enclosed, there is a $q \in \mathbb{Q}_{+}$with $q+\sum_{j \in \mathbb{N}}\left|I_{j}\right|<|J|$ and a sequence $\left(I_{j}^{\prime}\right)_{j \in \mathbb{N}}$ of intervals in $\mathbb{I}^{\mathbb{Q}}$ such that $I_{j} \subseteq I_{j}^{\prime}$ and $\left|I_{j}^{\prime}\right| \leq\left|I_{j}\right|+q 2^{-(j+1)}$ for all $j \in \mathbb{N}$, i.e. $\sum_{j \in \mathbb{N}}\left|I_{j}^{\prime}\right|$ converges to $q+\sum_{j \in \mathbb{N}}\left|I_{j}\right|$. In addition, there exists an interval $J^{\prime}$ in $\mathbb{I}^{\mathbb{Q}}$ such that $J^{\prime} \subseteq J$ and $q+\sum_{j \in \mathbb{N}}\left|I_{j}\right| \leq\left|J^{\prime}\right| \leq|J|$. Thus, a proof of Greenleaf's Theorem need only consider countably open sets (with sharp generators). Theorem 3.7 is hence a strengthening of Theorem 3.8.

As with non-sharp generators, a few standard results follow immediately from Greenleaf's Theorem and its generalization:

Corollary 3.9 (BISH) The following hold:

1. The set of reals in $[0 ; 1]$ cannot have an $\epsilon$-sharp cover with $\epsilon<1$.

2. For each function $f: \mathbb{N} \longrightarrow[0 ; 1]$, the set $\{x \in[0 ; 1]: x \neq f(n)$, for all $n \in \mathbb{N}\}$ is dense in $[0 ; 1]$, i.e. the set of reals in $[0 ; 1]$ is uncountable.

Proof. The first part is immediate. The second follows from the first part and Proposition 3.2 (for density, note that for all $J \in \mathbb{I}^{\mathbb{Q}}$ such that $J \subseteq[0 ; 1]$, we may choose a countably open set covering $f(\mathbb{N})$ with generator $\left(I_{j}\right)_{j \in \mathbb{N}}$ satisfying $\left.\sum_{j \in \mathbb{N}}\left|I_{j}\right|<|J|\right)$.

Corollary 3.10 (BISH) Let $A$ be the subset of the reals whose elements are limits of non-decreasing sequences of elements of $\mathbb{Q}$. For each countable subset $C \subseteq A$, there is an a $\in A$ such that $a \in A \backslash C$, i.e. $A$ is uncountable.

Pro of. By Theorem 3.7 and Proposition 3.2.

Close inspection of the proof of Theorem 3.7 reveals that we could have chosen the sequence to be strictly increasing instead of non-decreasing. We thus have:

Corollary 3.11 (BISH) Let $B$ be the subset of the reals whose elements are limits of strictly increasing sequences of elements of $\mathbb{Q}$. For each countable subset $C \subseteq B$, there is an $a \in A$ such that $a \in \mathbb{R} \backslash C$, i.e. $B$ is uncountable.

\section{Extensions of the Reals}

Given a non-sharp generator, there is in general no way of producing a real eventually avoiding the corresponding countably open set. However, the sequence of rationals produced by $M^{<}$when given such a generator is convergent within classical mathematics, whence sets of formal objects extending the (constructive) reals, e.g. Troelstra reals [Tro80, Ric98], Richman reals [Ric98], or fickle reals [Bis67, BB85] may contain the classical limit of the sequence. 
A fickle real is a bounded, non-decreasing sequence of elements of $\mathbb{Q}$. Since every bounded, nondecreasing (and, conversely, non-increasing) sequence in $\mathbb{Q}$ can be realised by considering a suitable generator, and conversely any generator defines a ficke real by the construction of Definition 3.1, the set of fickle reals constitute the only extension of the reals that we can hope to comment on using the material of the previous sections.

One may wonder whether there exists a bounded, countably open cover of the fickle reals in the style of Example 2.3.2. This turns out not to be the case:

Proposition 4.1 (BISH) For each $\epsilon$-enclosed countably open set there is a fickle real not covered by the set. If $\epsilon<1$, then there is a fickle real greater than 0 and less than 1 that is not covered by the set.

Proof. In both cases, if $\left(I_{j}\right)_{j \in \mathbb{N}}$ is a generator of such a countably open set, and $p, d \in \mathbb{Q}$ were arbitrary, Lemma 3.3 would yield that $M^{<}\left(p,\left(I_{j}\right)_{j \in \mathbb{N}}, d\right)$ would be a fickle real bounded away from $\bigcup_{j \in \mathbb{N}} I_{j}$. The bounds in the second part of the proposition also follow from the lemma.

Thus, the standard way of disproving the Heine-Borel theorem in RUSS is impossible for fickle reals. In computable analysis, the fickle numbers are equivalent to the left-computable reals [ZW01, Zhe02]. Thus, we could have substituted "fickle" with "left-computable" in Proposition 4.1. It is easy to see that the left-computable reals are countable within RUSS; observe that this creates no conflict with Proposition 3.2 since the proof of that proposition requires the considered objects to be reals.

The above propositions impose a hard limit on the techniques of this paper: we cannot hope to cover the fickle reals as we did $\mathbb{R}$ within RUSS. In the study of random numbers, P. Hertling and K. Weihrauch have shown in [HW98] that, using Martin-Löf's notion of randomness test [ML66], one can prove the existence of a random left-computable real by methods akin to those used in proving the existence of the cover of $\mathbb{R}$ in Example 2.3.2.

\section{Subsets of the Real Line Without Isolated Points}

Aberth was the first to demonstrate the existence of a sequence that is strongly Specker in [Abe80]; the lengthy proof was subsequently simplified by Bridges and Richman [BR87] using an elegant argument based on the very regular structure of the Cantor set. While the present paper so far has considered how to construct Specker sequences eventually bounded away from countably open sets, Bridges and Richman examine a particular set and show that it contains a Specker sequence.

This begs for generalization; we will be searching for a suitably general class of sets such that each set in the class contains a Specker sequence. It turns out that the following definition does the trick:

Definition 5.1 (BISH) A subset $A \subseteq \mathbb{R}$ is said to be without isolated points (abbreviated wip) if, for all $x \in A$ and all $\left.q \in \mathbb{Q}_{+},\right] x-q ; x+q[\cap A$ contains a point $y \neq x$. $A$ is called $\mathbb{Q}$-wip if $] x-q ; x+q[$ contains a point $y \neq x$ with $y \in \mathbb{Q}$.

In particular, the real line, any non-degenerate interval, and the Cantor set are all $\mathbb{Q}$-wip. The set of irrationals is wip, but not $\mathbb{Q}$-wip.

Recall from classical mathematics that a topological space is called perfect if it is wip and closed, and totally disconnected if the only connected subsets are the singletons. In classical topology, we have access to the celebrated Alexandroff-Hausdorff Theorem: The Cantor set is, up to homeomorphism, the unique nonvoid, totally disconnected, perfect, compact metrizable space [Wil70, HY88]. However, in constructive mathematics, we do not (yet) have that result available; even if we had, we would have to construct the homeomorphism in an order-preserving way if the target space was ordered in order to have strictly increasing sequences. Therefore, we will not attempt the full Alexandroff-Hausdorff Theorem, but instead show that every nonvoid, wip subset of the real line contains a subset having those topological properties of the Cantor set that ensure the existence of a strong Specker sequence within the subset.

Proposition $5.2(\mathrm{BISH})$ Let $P$ be a nonvoid, wip subset of $\mathbb{R}$. There is a sequence $\left(\mathcal{T}_{n}\right)_{n \in \mathbb{N}_{0}}$ of subsets of elements of $\mathbb{I}^{\mathbb{Q}}$, such that, for each $n \in \mathbb{N}_{0}$ :

1. $\mathcal{T}_{n}$ contains exactly $2^{n}$ intervals. 
2. If $I \in \mathcal{T}_{n}$ then $|I| \leq 3^{-n}$.

3. For each $I \in \mathcal{T}_{n}$ there are exactly two intervals $J, J^{\prime} \in \mathcal{T}_{n+1}$ such that $J, J^{\prime} \subseteq I$.

4. If $] p_{1} ; q_{1}[,] p_{2} ; q_{2}\left[\in \mathcal{T}_{n}\right.$ with $] p_{1} ; q_{1}[\neq] p_{2} ; q_{2}\left[\right.$, then either $q_{1}<p_{2}$ or $q_{2}<p_{1}$.

5. If $I \in \mathcal{T}_{n}$ there is an $x \in P \cap I$ such that if $] p_{1} ; q_{1}[,] p_{2} ; q_{2}\left[\in \mathcal{T}_{n+1}\right.$ with $] p_{1} ; q_{1}[,] p_{2} ; q_{2}[\subseteq I$, then $x<\min \left\{p_{1}, p_{2}\right\}$.

Pro of. We proceed by induction on $n$ :

- $n=0$. Since $P$ is nonvoid, there exists an element $x \in P$. Choose any $I \in \mathbb{I}^{\mathbb{Q}}$ such that $x \in I$ and $|I|=1$, and set $\mathcal{T}_{0} \triangleq\{I\}$. By wipness, there exists $y \in x \cap I$ with $y \neq x$, and the Hausdorff property of the real line allows us to find a neighborhood $U$ of $x$ such that $y \in P \backslash U$. By wipness, there is $z \in U \cap P$ with $z \neq x$, and we thus have three distinct points $x, y, z \in P \cap I$. Since the points are distinct, we may without loss of generality assume that $x<y<z$ and hence obtain disjoint $] p_{1} ; q_{1}[,] p_{2} ; q_{2}\left[\in \mathbb{I}^{\mathbb{Q}}\right.$ such that $\left.y \in\right] p_{1} ; q_{1}[, z \in] p_{2} ; q_{2}\left[\right.$, and such that $x<\min \left\{p_{1}, p_{2}\right\}$.

- $n>0$. By the induction hypothesis, it suffices to consider one interval $I \in \mathcal{T}_{n-1}$ and show that it contains two subintervals $I_{1}$ and $I_{2}$ with the desired properties. This is done exactly as in the proof of the base case.

Intuitively, the proposition means that we have an "infinite tree" of intervals, such that each interval has exactly two intervals as child nodes in the tree and contains a point of $P$ that is strictly less than all elements of the two child intervals $-\mathcal{T}_{n}$ is then the set of nodes of the tree at depth $n$.

Remark 5.3 (BISH) Since the construction of Proposition 5.2 is essentially an (infinite) full binary tree, we may consider the address, $\alpha$, of an interval and its associated point $x_{\alpha}$ as a finite binary sequence where "0" means "go left" in the tree, and " 1 " means "go right". The length of the address is denoted $|\alpha|$, and the empty sequence by $\lambda$ (with $|\lambda|=0$ and $I_{\lambda}$ being the single interval in $\mathcal{T}_{0}$ ).

Observe that since the $2^{n}$ intervals of $\mathcal{T}_{n}$ are pairwise separated by Proposition 5.2, there is, for each $n \in \mathbb{N}$, a $\delta_{n} \in \mathbb{Q}_{+}$such that the distance between the right endpoint of any interval in $\mathcal{T}_{n}$ and the left endpoint of any other interval in $\mathcal{T}_{n}$ is at least $\delta_{n}$. In addition, we have:

1. $x_{a_{1} \cdots a_{n}}<x_{a_{1} \cdots a_{n} \cdot 0}<x_{a_{1} \cdots a_{n} \cdot 1}$.

2. $x_{a_{1} \cdots 0 \cdots a_{n}}<x_{a_{1} \cdots 1 \cdots a_{n}}$ where the indicated '0' and '1' occur at identical indices.

3. $x_{a_{1} \cdots a_{n}}<x_{a_{1} \cdots a_{n} \cdot b_{1} \cdots b_{m}}$ for $m>0$.

Given an address $\alpha$, we may pick an $q_{\alpha} \in \mathbb{Q}$ such that $q_{\alpha}$ is the midpoint of the interval in $\mathcal{T}_{|\alpha|}$ having address $\alpha$. The following proposition establishes properties of the set of these $q_{\alpha}$ that we shall use as a stepping stone for the proof of the main result of this section.

Proposition 5.4 (BISH) Let $\{0,1\}^{\omega}$ be the set of infinite binary sequences. The following hold:

1. For each $\bar{\alpha} \in\{0,1\}^{\omega}$, write $\bar{\alpha}_{i}$ for the subsequence consisting of the least $i$ elements of $\bar{\alpha}$. Then, $q_{\bar{\alpha}} \triangleq\left(q_{\bar{\alpha}_{i}}\right)_{i \in \mathbb{N}_{0}}$ is a real number.

2. The set, A, consisting of all sequences $\left(q_{\bar{\alpha}_{i}}\right)_{i \in \mathbb{N}}$, is totally bounded, located, closed, and complete.

Proof. We proceed as follows:

1. By Proposition 5.2, the interval $I_{\alpha}$ with address $\alpha$ has width at most $3^{-|a|}$. If $I_{\beta}$ is an interval with address $\beta$ such that $\alpha$ is a prefix of $\beta$, then $I_{\beta} \subseteq I_{\alpha}$. Thus, $\left|q_{\bar{\alpha}_{j}}-q_{\bar{\alpha}_{j^{\prime}}}\right| \leq 3^{-|i|}$ for $j, j^{\prime}>i$. 
2. For each $n$, the set $\left\{q_{\alpha}:|\alpha|=n\right\}$ is a $3^{-n}$-approximation to $A$, showing that $A$ is totally bounded, and hence located. Observe that by Proposition 5.2, if $\left(x_{n}\right)_{n \in \mathbb{N}}$ is a convergent sequence of elements of $A$, the elements $x_{n}$ must eventually agree on the first $k \in \mathbb{N}$ digits of the address, for each $k$ (since the intervals of $\mathcal{T}_{n}$ are disjoint). Closure follows by standard methods, and completeness follows from closure.

We can now prove the first main result of this section:

Theorem 5.5 (RUSS) Every wip subset $P \subseteq \mathbb{R}$ contains a bounded, strictly increasing, strongly divergent sequence.

P r o of. Assume CPF and hence an enumeration, $\left(\phi_{m}\right)_{m \in \mathbb{N}}$, of all partial functions from $\mathbb{N}$ to $\mathbb{Q}$, and, for each $m \in \mathbb{N}$, an enumeration $D_{m}(1) \subseteq D_{m}(2) \subseteq \cdots$ of the domain $D_{m}$ of $\phi_{m}$, consisting of finite subsets of $\mathbb{N}$.

The set $K \triangleq\left\{m: m \in D_{m} \wedge \phi_{m}(m)=0\right\}$ is countable, since each element of the array

$$
\begin{array}{llll}
D_{1}(1) & D_{1}(2) & D_{1}(3) & \ldots \\
D_{2}(1) & D_{2}(2) & D_{2}(3) & \ldots \\
D_{3}(1) & D_{3}(2) & D_{3}(3) & \ldots \\
D_{4}(1) & D_{4}(2) & D_{4}(3) & \ldots
\end{array}
$$

can be "visited" by a standard zig-zag construction.

For future reference, we let $\iota: \mathbb{N} \longrightarrow K$ be an injective function enumerating $K$.

Let $w_{n}$ be the address having ' 0 's as its first $n-1$ bits and a single ' 1 ' as its $n$th digit. Define $\oplus$ as the bitwise 'or' operation on addresses, i.e. if $a$ and $b$ are addresses with, say, $|a| \geq|b|$, then $a \oplus b$ $(=b \oplus a)$ is the address defined by:

$$
\text { Bit } i \text { of } a \oplus b \triangleq\left\{\begin{array}{cl}
1 & \text { if } 1 \leq i \leq|b| \text { and bit } i \text { of either } a \text { or } b \text { or both is } 0 \\
0 & \text { if } 1 \leq i \leq|b| \text { and bit } i \text { of both } a \text { and } b \text { is } 0 \\
\text { bit } i \text { of } a & \text { if }|b|<i \leq|a|
\end{array}\right.
$$

Clearly, $\oplus$ is commutative and associative, and any finite $\oplus$-composition of $w_{n} \mathrm{~s}$ is an address, and by Proposition 5.4, each $q_{w_{n}}$ is a rational in the interval with address $w_{n}$ of the set $A$ of that proposition, and each $x_{w_{n}}$ is an element of $P$ occurring in the interval with address $w_{n}$ of the construction in Proposition 5.2.

It is a straightforward induction on $n$ to prove that

$$
x_{w_{\iota(1)} \oplus \cdots \oplus w_{\iota(n)} \oplus w_{\iota(n+1)}}>\max \left\{x_{w_{\iota(1)} \oplus \cdots \oplus w_{\iota(n)}}, x_{w_{\iota(n+1)}}\right\}
$$

; indeed, proceed as follows: By injectivity of $\iota$, the unique '1'-bits of $w_{\iota(i)}$ and $w_{\iota(j)}$ are in different places for $i \neq j$; hence adding $w_{\iota(n+1)}$ to $w_{\iota(1)} \oplus \cdots \oplus w_{\iota(n)}$ will either replace a '0' by a ' 1 ', or pad $w_{\iota(1)} \oplus \cdots \oplus w_{\iota(n)}$ with a finite number of '0's and a single '1'; the result then follows by the last part of Proposition 5.2. Also observe that injectivity of $\iota$ yields that if $N \geq m$ is so large that $m \in D_{m}(N)$, and if $n>N$, then bit $m$ of $w_{\iota(1)} \oplus \cdots \oplus w_{\iota(n)}$ is 1 iff $\phi_{m}(m)=0$.

Set $r_{n} \triangleq x_{w_{\iota(1)} \oplus \cdots \oplus w_{\iota(n)}}$. Observe that $\left(r_{n}\right)$ is strictly increasing by the above comments, and is bounded below by $q_{\lambda}-1$, and above by $q_{\lambda}+1$. Also note that the $\iota(k)$ th bit of the address of $r_{n}$ is identical for all $r_{n}$ if $n>k$.

The rest of the proof establishes that $\left(r_{n}\right)_{n \in \mathbb{N}}$ is strongly divergent.

Let $A$ be as in Proposition 5.4 and consider any $y \in A$. There exists an $m \in \mathbb{N}$ such that $\phi_{m}: \mathbb{N} \longrightarrow$ $\{0,1\}$ is total, and

$$
y=\left(q_{w_{\phi_{m}(1)} \oplus \cdots \oplus w_{\phi_{m}(n)}}\right)_{n \in \mathbb{N}}
$$


Choose $N \geq m$ so large that $m \in D_{m}(N)$. Then, as $\phi_{m}(m) \in\{0,1\}, \phi_{m}(m)=1-s_{n}(m)$ if $n \geq N$. Hence, by the final comments of Remark 5.3, $\left|y-r_{n}\right| \geq \delta_{m}$ for all $n \geq N$, completing the proof when $y \in A$.

Now, consider an arbitrary $y \in \mathbb{R}$. Recall that $A$ was located, and that the function $\rho_{A}(y)$ mapping $y$ to the infimum distance from $y$ to elements of $A$ is thus well-defined. Define an increasing binary sequence $\left(\lambda_{n}\right)_{n \in \mathbb{N}}$ such that, for each $n$, we have:

$$
\begin{aligned}
& \lambda_{n}=0 \Rightarrow \rho_{A}(y)<1 / n \\
& \lambda_{n}=1 \Rightarrow \rho_{A}(y)>1 /(n+1)
\end{aligned}
$$

Let $c$ be any point of $A$. If $\lambda_{1}=1$, define $a_{n} \triangleq c$ for all $n \in \mathbb{N}$; if $\lambda_{1}=0$, do the following for each $n>1$ :

- If $\lambda_{n}=0$, choose $a_{n} \in A$ with $\left|y-a_{n}\right|<1 / n$.

- if $\lambda_{n}=1$, set $a_{n} \triangleq a_{n-1}$.

Observe that $\left|a_{m}-a_{n}\right| \leq 2 / n$ if $m \geq n$. Thus, $\left(a_{n}\right)_{n \in \mathbb{N}}$ is Cauchy, hence-by Proposition 5.4converges to a limit, $a \in A$.

By the previous part of the proof, there exists $N \in \mathbb{N}$ and $\delta^{\prime} \in \mathbb{Q}_{+}$such that $\left|a-r_{n}\right| \geq \delta^{\prime}$ when $n \geq N$.

Now, either $|y-a|<\delta^{\prime}$, or $|y-a|>0$. In the first case, we have

$$
\left|y-r_{n}\right| \geq\left|a-r_{n}\right|-|y-a| \geq \delta^{\prime}-|y-a|
$$

whenever $n \geq N$. Choosing a $\delta \in \mathbb{Q}_{+}$with $\delta<\delta^{\prime}-|y-a|$ finishes the proof in this case.

In the second case, we may choose $m \in \mathbb{N}$ such that $\left|y-a_{m}\right| \geq 1 / m$; in this case, we have $\lambda_{m} \neq 0$, whence $\lambda_{m}=1$ and thus $\left.\rho_{A}(y)\right)>1 /(m+1)$. Setting $\delta \triangleq 1 /(m+1)$ furnishes $\left|y-r_{n}\right| \geq \rho_{A}(y) \geq \delta$ for all $n \in \mathbb{N}$, concluding the proof.

Finally, we have:

Theorem 5.6 (RUSS) Every $\mathbb{Q}$-wip subset of the reals contains a strong Specker sequence.

Proof. Identical to the proof of Theorem 5.5, except for the fact that the sequence can now be chosen to consist of elements of $\mathbb{Q}$.

\section{References}

[Abe80] O. Aberth. Computable Analysis. McGraw-Hill, 1980.

[BB85] E. Bishop and D. Bridges. Constructive Analysis, volume 279 of Grundlehren der mathematischen Wissenschaften. Springer-Verlag, 1985.

[BCSS97] L. Blum, F. Cucker, M. Shub, and S. Smale. Complexity and Real Computation. Springer-Verlag, 1997.

[Bis67] E. Bishop. Foundations of Constructive Mathematics. McGraw-Hill Series in Higher Mathematics. McGraw-Hill, 1967.

[BR87] D. Bridges and F. Richman. Varieties of Constructive Mathematics, volume 97 of London Mathematical Society Lecture Notes Series. Cambridge University Press, Cambridge, UK, 1987.

[HW98] P. Hertling and K. Weihrauch. Randomness Spaces. In Proceedings of ICALP'98, volume 1443 of Lecture Notes in Computer Science, pages 796 - 807. Springer-Verlag, 1998.

[HY88] J.G. Hocking and G.S. Young. Topology. Dover Publications, Inc., 1988.

[KL57] G. Kreisel and D. Lacombe. Ensembles récursivements mésurables et ensembles récursivements ouverts ou fermés. C.R. Acad. Sci. Paris, Ser. A-B, 245:1106-1109, 1057.

[Ko91] K.-I. Ko. Complexity Theory of Real Functions. Progress in Theoretical Computer Science. Birkhäuser, Boston, 1991.

[ML66] P. Martin-Löf. The Definition of Random Sequences. Information and Control, 9(6):602-619, 1966.

[Ric98] F. Richman. Generalized Real Numbers in Constructive Mathematics. Indagationes Mathematicae, 9:595-606, 1998. 
[Spe49] E. Specker. Nicht Konstruktiv beweisbare Sätze der Analysis. Journal of Symbolic Logic, 14:145-158, 1949.

[Spe59] E. Specker. Der Satz vom Maximum in der rekursiven Analysis. In A. Heyting, editor, Constructivity in Mathematics, pages 254-265. North-Holland, 1959.

[Tro80] A.S. Troelstra. Intuitionistic Extensions of the Reals. Nieuw Archief voor Wiskunde, 28:63-113, 1980.

[TZ62] G.S. Tseitin and I.D. Zaslavskij. On Singular Coverings and Properties of Constructive Functions Connected with them. Tr. mat. Inst. Steklov 67, 458-502 (translated in Amer. Math. Soc., Transl., II Ser. 98, 41-89), 1962.

[Wei98] K. Weihrauch. Computable Analysis: An Introduction. Springer, 1998.

[Wil70] S. Willard. General Topology. Addison-Wesley, 1970.

[Zhe02] X. Zheng. Recursive Approximability of Real Numbers. Mathematical Logic Quarterly, 48:131-156, 2002.

[ZW01] X. Zheng and K. Weihrauch. The Arithmetical Hierarchy of Real Numbers. Mathematical Logic Quarterly, 47(1):51-65, 2001. 